\title{
Clinical antiarrhythmic effects of amiodarone in patients with resistant paroxysmal tachycardias
}

\author{
D E WARD, A J CAMM, R A J SPURRELL \\ From the Cardiology Department, St Bartholomew's Hospital, West Smithfield, London
}

SUMMARY Oral amiodarone, an iodine-containing antiarrhythmic agent, was administered to 72 patients with recurrent paroxysmal tachycardias. Thirty-nine patients had tachycardias associated with the Wolff-Parkinson-White syndrome, 15 patients had paroxysmal atrial fibrillation unassociated with the Wolff-Parkinson-White syndrome, and 18 patients had ventricular tachycardia. In all patients, the frequency of symptomatic attacks had not been reduced by at least three other antiarrhythmic agents alone or in combination. The response to amiodarone treatment was graded according to the patients' subjective response (total suppression, partial suppression, and no effect). Overall, 57 per cent of patients had total abolition of attacks and another 22 per cent had a partial suppression of attacks. Side effects, the most common of which were photosensitivity and gastrointestinal upsets, occurred in 44 per cent and were sufficiently severe to warrant withdrawal of treatment in 15 per cent. These results confirm that amiodarone is of considerable value in the treatment of recurrent paroxysmal arrhythmias resistant to other drugs.

Amiodarone, a benzfuran derivative, is an antiarrhythmic drug which was originally used in Europe as an antianginal agent. ${ }^{1}$ Singh and Vaughan-Williams ${ }^{2}$ subsequently demonstrated that the drug prolonged the action potential. Intracardiac electrophysiological studies in man indicated that oral amiodarone increased the refractoriness of atrial myocardium, atrioventricular node, ventricular myocardium, and anomalous bypass tracts. ${ }^{3}$ Recent clinical studies ${ }^{4-7}$ have shown the beneficial effect of the drug in a variety of arrhythmias, the majority of which were refractory to several other antiarrhythmic agents. These studies indicate that amiodarone is an extremely effective antiarrhythmic agent and forms an important addition to the therapeutic armamentarium. As yet, in this country, the drug has no clinical trials certificate and can only be prescribed for specified named patients.

We report our experience of the use of oral amiodarone in 39 patients with tachyarrhythmias associated with the Wolff-Parkinson-White (WPW) syndrome, 15 patients with paroxysmal atrial fibrillation or flutter unassociated with the WPW syndrome, and 18 patients with recurrent paroxysmal ventricular tachycardia.

\section{Patients and methods}

The patients, aged 12 to 73 years, had distressing paroxysmal palpitation repeatedly documented electrocardiographically, which had not been symptomatically improved by any other antiarrhythmic drugs. All patients had previously been treated with at least three other antiarrhythmic drugs in conventional doses. The details of the patients and their arrhythmias are summarised in Table 1. Electrophysiological studies were performed in all patients with accessory pathways to define the tachycardia mechanism and the characteristics of the accessory pathway. Recurrent paroxysmal atrial fibrillation or flutter and ventricular tachycardia were diagnosed from 24-hour ambulatory recordings or from 12 lead electrocardiograms taken during the attack. Patients with ventricular tachycardia were subdivided on the basis of the coronary arteriographic and left ventricular angiographic findings.

Oral amiodarone was started using a loading dose of $600 \mathrm{mg}$ daily for one week, $400 \mathrm{mg}$ daily for one week, and a subsequent dosage of at least $200 \mathrm{mg}$ daily adjusted according to the response. Amiodarone was not continued for more than four weeks if there was no therapeutic response. 
Table 1 Results

\begin{tabular}{|c|c|c|c|c|}
\hline Response & Total & Partial & $\mathrm{Nil}$ & Total (n) \\
\hline \multicolumn{5}{|c|}{$\begin{array}{l}\text { Wolff-Parkinson-White ( } 39 \text { patients) * } \\
\text { Paroxysmal atrial }\end{array}$} \\
\hline fibrillation & 4 & 1 & 1 & 6 \\
\hline \multicolumn{5}{|c|}{$\begin{array}{l}\text { Paroxysmal atrial } \\
\text { fibrillation/supraventricular }\end{array}$} \\
\hline tachycardia & 7 & 1 & 2 & 10 \\
\hline \multicolumn{5}{|l|}{ Supraventricular } \\
\hline tachycardia & 12 & 7 & 4 & 23 \\
\hline Total & 23 & 9 & 7 & 39 \\
\hline \multicolumn{5}{|c|}{ Paroxysmal atrial futter or fibrillation (15 patients) $\dagger$} \\
\hline Fibrillation & 6 & 3 & 2 & 11 \\
\hline Flutter & 2 & 1 & 1 & 4 \\
\hline Total & 8 & 4 & 3 & 15 \\
\hline \multicolumn{5}{|c|}{ Ventricular tachycardia (18 patients) $\ddagger$} \\
\hline $\begin{array}{l}\text { Ischaemic } \\
\text { (Acute myocardial }\end{array}$ & 8 & 1 & 3 & 12 \\
\hline infarction) & (3) & 1 & 一 & 4) \\
\hline Cardiomyopathy & 1 & 2 & 1 & 4 \\
\hline Unknown cause & 1 & - & 1 & 2 \\
\hline Total & 10 & 3 & 5 & 18 \\
\hline
\end{tabular}

$\star$ Type $A=25$, Type $B=3$, Concealed $=11$, male $n=20$, female $n=19$.

† Male, 10 ; female, 5 . $\ddagger$ Male $n=13$, female $n=5$.

Intermittent 24-hour ambulatory monitoring is not a practical method of assessment in patients with relatively infrequent arrhythmias occurring over a long period of time. For this reason we chose to grade the response to treatment according to the frequency of subjective symptoms rather than objective ambulatory electrocardiographic findings which, because of the intermittent nature of arrhythmias, are often difficult to interpret. In addition, only patients with sustained symptomatic paroxysms of tachycardia were selected. Patients with ectopic beats only were excluded because these arrhythmias are more difficult to relate to symptoms.

The response to treatment was therefore graded into three categories according to the patient's subjective assessment: total suppression of attacks: no awareness of further attacks; partial suppression: patient aware of less frequent and less troublesome attacks; no demonstrable effect: the patient reported no improvement. These criteria are similar to those of Rosenbaum et al. ${ }^{5}$

Follow-up has ranged from three months to five years. Thyroid function tests, liver function tests, full blood count, electrolytes, and urea were performed at regular intervals during follow-up. The presence or absence of corneal deposits ${ }^{8}$ and other unwanted effects were noted at each visit.

\section{Results}

The overall results in each category of arrhythmia are shown in Table 1. (a) WOLFF-PARKINSON-WHITE TACHYCARDIAS Of the 39 patients with symptomatic tachycardias associated with the WPW syndrome, all of whom had failed to respond to disopyramide, quinidine, beta blockers, or verapamil, $23(59 \%)$ had total abolition of attacks. The majority of these patients were suffering from atrioventricular re-entrant tachycardia. The maintenance dose in 17 of these 23 patients was $200 \mathrm{mg}$ daily and only one patient required more than $600 \mathrm{mg}$ daily (Table 2). Nine patients had partial suppression and seven patients failed to notice any symptomatic improvement despite higher maintenance doses.

(b) PAROXYSMAL ATRIAL FIBRILLATION OR FLUTTER UNASSOCIATED WITH WPW SYNDROME

All the patients in this group had been unsuccessfully treated with disopyramide, quinidine, digoxin, verapamil, or beta blockers. Of the total of 15 patients, total suppression was achieved in eight patients, two of whom had paroxysmal atrial flutter. In six of these eight patients the maintenance dose of $200 \mathrm{mg}$ daily was sufficient to control the arrhythmia and none required more than $400 \mathrm{mg}$ daily. None of these patients had evidence of coronary artery disease or any other disorder which may have predisposed to the development of atrial fibrillation. There was no evidence of sinus node dysfunction before amiodarone treatment in any patient in this group.

\section{(c) VENTRICULAR TACHYCARDIA}

In 12 patients there was evidence of coronary artery disease (previous myocardial infarction in nine patients) confirmed by coronary arteriography. Six patients had abnormal left ventricular angiograms with evidence of aneurysm formation in four patients. Six patients had normal coronary arteries

Table 2 Therapeutic effect versus maintenance dose

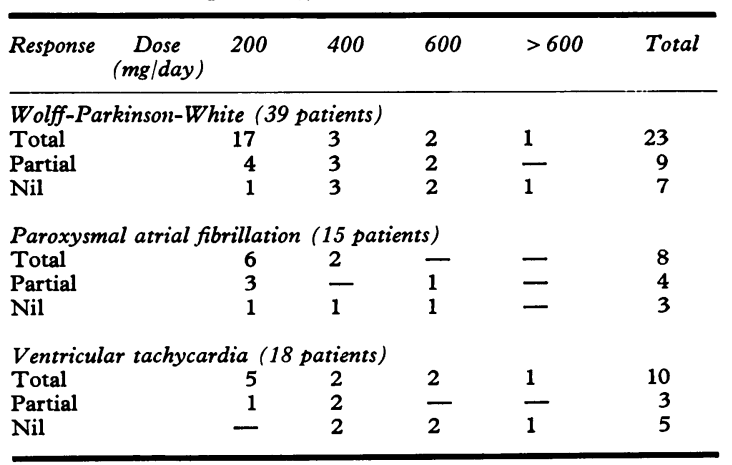


and four of these were considered to have a cardiomyopathy. In two patients the underlying aetiology of tachycardia was unknown. No patient had ventricular fibrillation. All patients were resistant to other antiarrhythmic agents which included the class I drugs disopyramide, mexiletine, and phenytoin. ${ }^{9}$ Eight patients also had beta blocking agents without benefit. Four patients were started on amiodarone in the immediate post-infarction period after they had failed to respond to lignocaine and disopyramide. An excellent response was observed in three of these patients. Overall, total suppression was obtained in 10 of the 18 patients.

There appeared to be no apparent distinguishing characteristics of those in whom total suppression was achieved. For example, age, sex, location of accessory pathway in patients with the WPW syndrome, type of arrhythmia, or aetiology of ventricular tachycardia did not seem to influence the response to treatment. The duration of onset of action ranged from two days to three weeks. Of the poor responders with the WPW syndrome, four subsequently underwent cryosurgical ablation of the accessory pathway. Surgical treatment was also undertaken in two patients with the WPW syndrome who were intolerant to the drug despite an excellent response. This was also the case in two patients with partial suppression of paroxysmal atrial fibrillation, who, together with two others with no response, underwent surgical His bundle ablation. One patient with ventricular tachycardia of unknown aetiology eventually required surgical ablation of the abnormal focus, after failure to respond to amiodarone. Side effects other than corneal deposits occurred in 44 per cent of patients and included gastrointestinal upsets (14\%), photosensitivity $(17 \%)$, nightmares and vivid dreams $(10 \%)$, headaches $(7 \%)$, paraesthesiae $(6 \%)$, epistaxis in one patient, and profound sinus bradycardia in two patients. Side effects necessitated discontinuation of treatment in 11 patients (gastrointestinal upset, four patients; nightmares, three patients; headaches, two patients; photosensitivity, two patients). The two patients with sinus bradycardia (WPW, one patient, atrial flutter, one patient) were treated by permanent pacing. Corneal deposits were observed in 51 patients at three months and in 43 of the 46 patients still on treatment at six months. In no patient did these deposits interfere with vision.

Most patients showed increases in T4 and free thyroxine index with normal thyroid hormone uptake tests but none developed clinical thyroid disease; these results are reviewed in detail elsewhere. ${ }^{10}$ There appeared to be no relation between biochemical disturbance of thyroid function and antiarrhythmic efficacy. Abnormal liver function was noted in one patient who was subsequently found to have coincidental liver disease. No abnormalities of renal function or full blood count were observed.

\section{Discussion}

The major cellular electrophysiological effect of amiodarone is pronounced prolongation of action potential duration ${ }^{2}$ with little effect on the rate of rise of the upstroke. In this respect it differs from other antiarrhythmic agents with the exception of sotalol. ${ }^{11}$ Previously reported clinical studies ${ }^{4-7}$ had shown the drug to be extremely effective in controlling a wide variety of recurrent tachyarrhythmias.

Rosenbaum et al..$^{5}$ noted total suppression in all 27 patients with tachyarrhythmias related to the WPW syndrome. This compares with 59 per cent of the current series though another 23 per cent had a good response. This difference may be accounted for by the fact that not all the patients reported by Rosenbaum et al. ${ }^{5}$ had had previous treatment with three or more antiarrhythmic agents and may have been less resistant to medical treatment than our patients. The maintenance dose of amiodarone required to obtain total suppression, however, was greater than $300 \mathrm{mg}$ daily in nine of 11 patients in Rosenbaum et al.'s initial series, ${ }^{4}$ whereas only six of our 23 patients with total suppression required more than $200 \mathrm{mg}$ daily. Differences in patient selection may also have contributed to the discrepancy between our results in this group and those of Rosenbaum et al. ${ }^{5}$ If only the patients with paroxysmal atrial fibrillation associated with the WPW syndrome are considered, the success rate in terms of total abolition of attacks was 69 per cent. It is unlikely that the presence of this arrhythmia made any difference to the observed response. The proportion of patients with paroxysmal atrial fibrillation in our series is comparable to that of Rosenbaum's series.

Total suppression of attacks was obtained in a smaller porportion of patients $(53 \%)$ with paroxysmal atrial fibrillation or flutter unassociated with the presence of an accessory pathway. This compares with 96 per cent of a series of 30 patients reported by Rosenbaum ${ }^{5}$ which included seven patients with the WPW syndrome. Almost all of the remainder of these patients, however, had associated cardiovascular or pulmonary disease in contrast to our patients who were otherwise apparently healthy. In particular, no patient had evidence of sinus node disease on 24-hour ambulatory recordings, though one patient developed profound sinus bradycardia while on amiodarone 
which is known to depress sinus automaticity. ${ }^{2} 12$ This side effect also occurred in one patient with the WPW syndrome and both patients were successfully treated by implantation of a permanent pacemaker.

The success of amiodarone in suppressing recurrent ventricular arrhythmias has been documented previously ${ }^{5-7}$ in patients with ischaemic heart disease and in those with cardiomyopathy. Of Rosenbaum's series of $\mathbf{4 4}$ patients including 13 with evidence of coronary artery disease, total suppression of attacks was achieved in $34(77 \%)$. Of our 12 patients with ischaemic heart disease, eight exhibited total suppression, including three patients with arrhythmias in the acute postmyocardial infarction period. Indeed, previous experience, though limited, ${ }^{56}$ suggests that the drug is no less effective in the suppression of ventricular arrhythmias related to acute ischaemia. This beneficial effect is probably related to several actions of amiodarone. In experimental animals the drug has been shown to produce coronary vasodilatation,,$^{1314}$ to increase coronary flow,,$^{14}$ to decrease left ventricular work, and to decrease left ventricular oxygen consumption. ${ }^{13} 14$ These effects, by tending to relieve ischaemia, would be expected to have a beneficial effect on ventricular tachycardias related to ischaemia.

Amiodarone, however, also has several direct effects. For example, the intravenous administration of the drug to experimental animals has an acutely protective effect against the development of ventricular arrhythmias induced by ouabain, ${ }^{2}$ aconitine crystals, ${ }^{15}$ and coronary artery ligation. ${ }^{.15}$ It is not known whether these effects are related to the cellular actions of the drug. Chronic oral administration has also been shown to be effective in suppressing ventricular arrhythmias induced by coronary ligation ${ }^{5}$ and under these circumstances the cellular effects of amiodarone, which are known to be pronounced after chronic administration, ${ }^{25}$ may be of some importance. In man, chronic amiodarone administration increases the ventricular effective refractory period. ${ }^{3}$ The relevance of this observation to the therapeutic effect of the drug is, however, unknown. In addition, there is no evidence to suggest that the effectiveness of amiodarone in atrial arrhythmias is simply related to prolongation of the atrial effective refractory period. On the other hand, prolongation of accessory pathway refractoriness is likely to be an important effect in the suppression of sustained atrioventricular reentrant tachycardia associated with the WPW syndrome ${ }^{3}$ but it cannot be assumed that this is the only factor involved. It is probable that the antiarrhythmic action of amiodarone is a result of the complex interaction of several effects, including suppression of ectopic impulses which may initiate tachycardia. ${ }^{16} 17$ The relation of amiodarone-induced disturbances of thyroid function to observed clinical antiarrhythmic effects is not known. Singh and Vaughan-Williams ${ }^{2}$ showed that the cellular effects of chronic amiodarone administration could be countered by simultaneous treatment with thyroxine. The precise mechanism of the biochemical disturbance is ill understood, however, and we have as yet not noted any relation between such disturbances and the antiarrhythmic efficacy of the drug. In any case it is unlikely that a "thyroid" effect could explain the rapid onset of action of the drug in a proportion of cases.

The incidence of side effects in our series is relatively high when compared with Rosenbaum's series, ${ }^{5}$ though the commonest unwanted effects (gastrointestinal upset and photosensitivity) occurred in roughly the same proportion of patients in each study. The incidence of side effects necessitating withdrawal of amiodarone was much higher than that reported by Rosenbaum. ${ }^{5}$ The reasons for this discrepancy are not clear, but do not appear to be related to the average maintenance dose or duration of treatment which are comparable in the two series. Corneal deposits were asymptomatic in all patients in whom they occurred, and we believe that this effect, which is reversible, ${ }^{8}$ is not as serious as other authors have suggested..$^{56}$

Although we agree with the principle of Rosenbaum's contention that the need for surgery is considerably diminished by the availability of amiodarone it is evident that in a small number of patients refractory to the drug surgical intervention has an important role.

In total, a beneficial therapeutic response was obtained in 79 per cent of patients. These results, though not as good as those obtained by some investigators, ${ }^{45}$ clearly confirm early results ${ }^{18}$ suggesting that amiodarone is of great value in the suppression of a variety of paroxysmal arrhythmias resistant to other antiarrhythmic drugs. The relatively high incidence of side effects is disappointing, however, and may limit the overall usefulness of the drug.

\section{References}

1 Vastesaeger M, Gillet P, Rasson G. Étude clinique d'une nouvelle medication antiangoreuse. Acta Cardiol (Brux) 1967; 22: 483-500.

2 Singh BN, Vaughan-Williams EM. The effect of amiodarone, a new anti-anginal drug, on cardiac muscle. $\mathrm{Br}$ f Pharmacol 1970; 39: 657-67.

3 Wellens HJJ, Lie KI, Bär FW, et al. Effect of amio- 
darone in the Wolff-Parkinson-White syndrome. Am f Cardiol 1976; 38: 189-94.

4 Rosenbaum M, Chiale PA, Ryba D, Elizari MV. Control of tachyarrhythmias associated with WolffParkinson-White syndrome by amiodarone hydrochloride. Am $\mathcal{F}$ Cardiol 1974; 34: 215-23.

5 Rosenbaum M, Chiale PA, Halpern MS, et al. Clinical efficacy of amiodarone as an antiarrhythmic agent. Am F Cardiol 1976; 38: 934-44.

6 Leak D, Eydt JN. Control of refractory cardiac arrhythmias with amiodarone. Arch Intern Med 1979; 139: 425-8.

7 Wheeler PJ, Puritz R, Ingram DV, Chamberlain DA. Amiodarone in the treatment of refractory supraventricular and ventricular arrhythmias. Postgrad Med F 1979; 55: 1-9.

8 Vérin PH, Gendre PH, Barchewitz G, LaurentBronchat G, Yacoub M, Morax S. Thesaurismose cornéenne par l'amiodarone. Données récentes. Arch Ophtalmol (Paris) 1971; 31: 581-96.

9 Vaughan-Williams EM. Classification of antidysrhythmic drugs. Pharmacol Ther Bull 1975; 1: 115-38.

10 Rees L, Ward DE, Al-Hamdi A, Camm AJ. Effect of amiodarone on thyroid function. International congress and symposium series. no. 16. London: Royal Society of Medicine, 1980; 53-6.

11 Singh BN, Vaughan-Williams EM. A third class of antiarrhythmic action. Effects on atrial and ventri- cular intracellular potentials, and other pharmacological actions on cardiac muscle, of MJ1999 and AH3474. Br f Pharmacol 1970; 39: 675-87.

12 Touboul P, Porte J, Huerta F, Delahaye JP. Electrophysiological effects of amiodarone in man (abstract). Am $\mathcal{F}$ Cardiol 1975; 35: 173.

13 Charlier R, Deltour G, Baudine A, Chaillet F. Pharmacology of amiodarone, an anti-anginal drug with a new biological profile. Arzneim Forsch 1968; 11: 1408-17.

14 Petta JM, Zaccheo VJ. Comparative profile of L3428 and other antianginal agents on cardiac hemodynamics. F Pharmacol Exp Ther 1971; 176: 328-38.

15 Charlier R, Deltour G. Correction des arythmies expérimentales par l'amiodarone. $\mathcal{F}$ Pharmacol (Paris) $1970 ; 1: 175-82$.

16 Tadei A, Richard M, Victor J, Delhumeau A, Geslin P. Étude de l'amiodarone dans les troubles du rythme ventriculaire. Arch Med Ouest 1974; 6: 41534.

17 Van Schepdael J, Solvay H. Étude clinique de l'amiodarone dans les troubles du rythme cardiaque. Presse Med 1970; 78: 1849-50.

18 Coumel P, Bouvrain Y. Étude clinique des effets pharmacodynamiques et antiarythmiques de l'amiodarone. F Agrégés 1973; 6: 69-81.

Requests for reprints to Dr D E Ward, Brompton Hospital, Fulham Road, London SW3 6HP. 